\title{
On the adhesion-velocity relation and length adaptation of motile cells on stepped fibronectin lanes
}

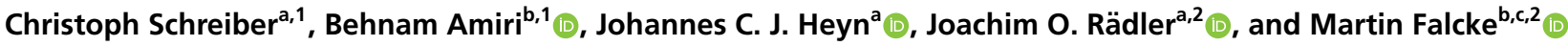 \\ ${ }^{a}$ Faculty of Physics and Center for NanoScience, Ludwig-Maximilians-Universität München, 80539 Munich, Germany; ${ }^{b}$ Max Delbrück Center for Molecular \\ Medicine in the Helmholtz Association, 13125 Berlin, Germany; and 'Department of Physics, Humboldt University, 12489 Berlin, Germany
}

Edited by Yu-li Wang, Carnegie Mellon University, Pittsburgh, PA, and accepted by Editorial Board Member Yale E. Goldman December 7, 2020 (received for review May 23, 2020)

The biphasic adhesion-velocity relation is a universal observation in mesenchymal cell motility. It has been explained by adhesionpromoted forces pushing the front and resisting motion at the rear. Yet, there is little quantitative understanding of how these forces control cell velocity. We study motion of MDA-MB-231 cells on microlanes with fields of alternating Fibronectin densities to address this topic and derive a mathematical model from the leading-edge force balance and the force-dependent polymerization rate. It reproduces quantitatively our measured adhesion-velocity relation and results with keratocytes, PtK1 cells, and $\mathrm{CHO}$ cells. Our results confirm that the force pushing the leading-edge membrane drives lamellipodial retrograde flow. Forces resisting motion originate along the whole cell length. All motion-related forces are controlled by adhesion and velocity, which allows motion, even with higher Fibronectin density at the rear than at the front. We find the pathway from Fibronectin density to adhesion structures to involve strong positive feedbacks. Suppressing myosin activity reduces the positive feedback. At transitions between different Fibronectin densities, steady motion is perturbed and leads to changes of cell length and front and rear velocity. Cells exhibit an intrinsic length set by adhesion strength, which, together with the length dynamics, suggests a spring-like front-rear interaction force. We provide a quantitative mechanistic picture of the adhesion-velocity relation and cell response to adhesion changes integrating force-dependent polymerization, retrograde flow, positive feedback from integrin to adhesion structures, and spring-like front-rear interaction.

cell motility | cell adhesion | cellular biophysics | surface micropattern

ell motility is crucial for various processes, ranging from - migration of cells in development and tumor metastasis to neuronal growth-cone advance in the formation of neuronal connectivity (1-3). Many cells form a large, thin protrusion in the direction of motion when moving on flat substrates (4). The whole protrusion is mechanically stabilized by adhesion with the substrate (5-10). Treadmilling of a dense network of branched actin filaments (F-actin) inside it pushes the leadingedge membrane forward (11-13). That generates motion, since the filament barbed ends polymerize at the leading edge of the lamellipodium and, thus, maintain the protrusion force (1417). Each added monomer extends the filament by the length $d=2.7 \mathrm{~nm}$. Further back, the pointed ends depolymerize, and filaments are severed $(12,13)$.

The F-actin network moves relative to the protrusion's leading edge due to polymerization $(11,18)$. The velocity of this retrograde flow is the network-extension rate $v_{e}$ in the cell frame of reference and the vectorial difference of $v_{e}$ and cell velocity $v$ in the laboratory frame of reference. This flow causes friction with all structures relative to which it moves, in particular, also with the intracellular interface of adhesion structures and stress fibers (19-22).

The F-actin network transmits the protrusion force via adhesion sites and adhesive forces to the substrate (23-25). Studies on network flow $(19,21,22)$ and measurements of the dynamic force-velocity relation (16) showed that the protrusion force is transmitted to adhesion structures by friction between the flowing F-actin network and these structures, and not by a direct elastic connection between leading-edge membrane and adhesion sites.

Retrograde flow is the fastest in the lamellipodium subregion of the F-actin network directly at the leading-edge membrane. It substantially slows down at the transition to the lamella region, which adjoins the lamellipodium (26-29). Nascent focal adhesion (FA) sites start to emerge under the lamellipodium and mature toward the lamella $(20,25,27,30)$. The boundary between the lamella and the lamellipodium coincides with an elevated density of FAs (20). Retrograde flow slows down at these FAs (20), relative tension gradients are large (24), and velocity gradients get steeper with increasing adhesion density $(20,31)$. These observations illustrate directly the friction between the flowing network and stationary structures.

The density of adhesion-related structures and strength of adhesion can be controlled experimentally by the substrate density of the ligand (e.g., Fibronectin) of the adhesion molecule, which is integrin most of the time $(6-9,31-34)$. The percentage of cell ventral area covered by adhesion structures increases with Fibronectin density in PtK1 cells (31). Varying Fibronectin density led to the discovery of the biphasic dependency of the cell velocity on ligand density, which is a fundamental and universal experimental observation in this context $(6-9,31-33,35)$.

\section{Significance}

Cells exert forces on their environment by contracting actin networks, friction of intracellular F-actin flow, and polymerization when they move, e.g., during tumor metastasis or development. In this context, the relation between adhesion and cell velocity is a general cell-type-independent observation, the investigation of which bears the chance of understanding basic mechanisms. Restricting cell motion to one-dimensional lanes simplifies the problem and allows for comparison to mathematical models. Polymerization at the cell's leading edge drives F-actin network flow and pushes the membrane. The drag of detaching the cell, the membrane, and the cell body resist motion. Since only velocitycontrolled forces shape motion, cells can move even across highly adhesive areas without getting stuck.

Author contributions: J.O.R. and M.F. designed research; C.S., B.A., and J.C.J.H. performed research; C.S. and B.A. analyzed data; and C.S., B.A., J.O.R., and M.F. wrote the paper. The authors declare no competing interest.

This article is a PNAS Direct Submission. Y.-I.W. is a guest editor invited by the Editorial Board.

This open access article is distributed under Creative Commons Attribution-NonCommercialNoDerivatives License 4.0 (CC BY-NC-ND)

${ }^{1}$ C.S. and B.A. contributed equally to this work

${ }^{2}$ To whom correspondence may be addressed. Email: raedler@Imu.de or martin.falcke@ mdc-berlin.de.

This article contains supporting information online at https://www.pnas.org/lookup/suppl/ doi:10.1073/pnas.2009959118/-/DCSupplemental.

Published January 22, 2021. 
The existence of a velocity maximum in dependence on adhesion has been explained by the action of adhesion on both cell front and rear $(6-10,24,31,32,36)$. The velocity increases initially with adhesion strength, since pushing force at the front can be transmitted better to the substrate. Moving cells need to pull the rear membrane off the adhesion bonds, which causes resistance to motion and decreases velocity with increasing adhesion.

The initial hypothesis on the mechanism of front-rear interaction was graded adhesion allowing for pulling off rear adhesions with force from the front transmitted by stress fibers, since adhesions at the front were assumed to be stronger than those at the rear $(37,38)$. Detailed experimental analysis revealed complex feedbacks between adhesion and intracellular force generation and could not directly confirm the graded adhesion mechanism $(20,31,33)$. It remained unresolved whether adhesion at the front needs to be stronger than at the rear and where forces resisting motion actually originate. As yet, there is little mechanistic and quantitative understanding of effects of adhesion on front versus rear regions of the cell, or front-rear interaction required for the suggested mechanism of the biphasic relation to hold. To resolve this issue, we need to measure steady-state cell velocities and length at different adhesion strengths; study the dynamic adaptation at adhesion transitions, where front and rear transiently experience different ligand densities; and compare results to force models.

In the present study, we restricted cell motility to onedimensional motion, which substantially simplifies the analysis by avoiding shape changes occurring on two-dimensional substrates (Movie S1) (33). In addition, since heterogeneities within cell populations easily obscure weak effects of adhesion, we subjected individual cells to steps of adhesion strength and measured relative velocity changes of single cells. Micropatterning techniques like microcontact printing (39) have been used to confine cell migration to protein-coated one-dimensional lanes (40-42) or to impose defined cell shapes (43-46). However, so far, micropatterns with variations of protein coating within the pattern (47) have not been used to study the velocity and length adaptation of cells to stepwise variations of adhesion strength.

We investigated the mechanism of the biphasic adhesionvelocity relation and the character of the front-rear interaction by studying motion of cells on Fibronectin lanes fabricated with two-step microcontact printing. We started with recapitulating the biphasic adhesion-velocity relation for MDA-MB-231 cells. The force balance at the leading edge and force dependency of polymerization allow derivation of a mathematical model and, thus, for quantitatively analyzing our results and also published data for keratocytes, PtK1 cells, and CHO cells. This quantitative analysis provides mechanistic insight into the biphasic relation. The stationary case with cell front and back moving on homogeneous Fibronectin density with the same velocity is not sufficient to investigate the coupling between front and rear. Therefore, we perturbed motion by alternating Fibronectin density steps and analyzed the dynamics of cells during transition across the density interfaces. This provides insight into the adhesion-length relation and the character of the force mediating front-rear interaction.

\section{Results}

The Velocity of MDA-MB-231 Cells on Microlanes Shows Biphasic Dependence on Fibronectin Density. We developed a microcontact printing protocol for Fibronectin-coated microlanes with varying density that is based on two stamping steps (SI Appendix, Fig. S1). It created $15-\mu \mathrm{m}$-wide lanes with alternating fields of specific Fibronectin densities. The area between the microlanes was coated with cell-repellent polyethylene glycol (PEG) (Fig. 1A). The Fibronectin absolute surface density was estimated by the fluorescence intensity of the labeled Fibronectin for each segment individually (SI Appendix, Fig. S2). MDA-MB-231 breast
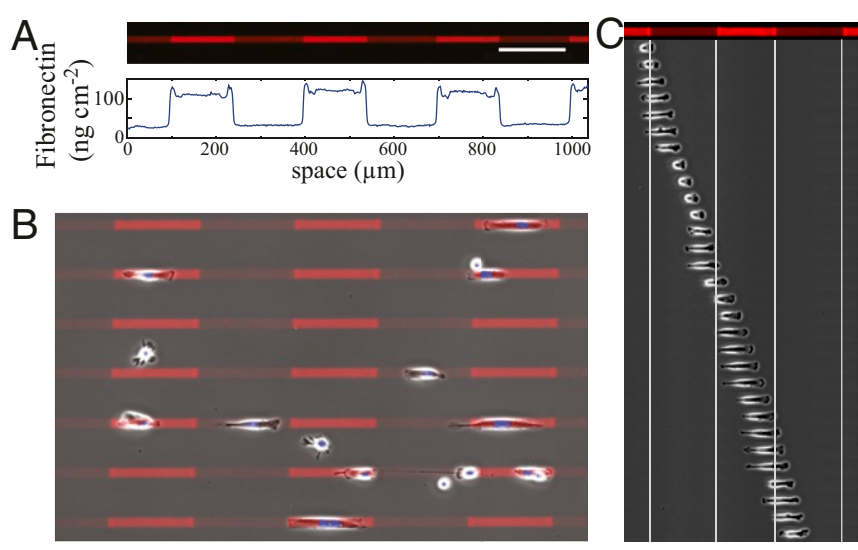

Fig. 1. Fibronectin lanes and cell motion. (A) Fluorescence image of a Fibronectin-coated lane with fields of different Fibronectin density shown below. (Scale bar: $150 \mu \mathrm{m}$.) (For a discussion of absolute Fibronectin densities, see SI Appendix, section S1B.) (B) MDA-MB-231 cells seeded on Fibronectin lanes. Overlay of phase-contrast and fluorescence images of patterns (red) and nuclei labeled with Hoechst (blue). Cells are restricted to one-dimensional motion on the microlanes and frequently traverse to fields with different Fibronectin densities. They sometimes also spontaneously reverse direction or stay at one position for some time. (C) Time course of the position of a single cell migrating on the lane shown on top. Phase-contrast images were taken every $10 \mathrm{~min}$.

cancer cells were seeded on the lanes (Fig. $1 B$ and $C$; Movie $\mathrm{S} 2$ ). The width of the lanes of $15 \mu \mathrm{m}$ is still broad enough for the morphology of the cells being close to the morphology in two dimensions (40), but major protrusions can only form along the direction of the lane (Movie S1). The cell nuclei were fluorescently labeled to allow automated cell tracking (Fig. $1 A$ ). We used scanning time-lapse microscopy and were able to observe about 2,000 single cells in about 100 view fields per measurement. Images were taken every $10 \mathrm{~min}$ for $48 \mathrm{~h}$.

We used lanes with different combinations of Fibronectin densities and analyzed more than 15,000 single-cell tracks. The velocity averaged over the cell population shows the biphasic behavior with maximal velocity for intermediate Fibronectin densities (Fig. $2 B$ ) that was reported by several studies for different cell types $(6-9,31,33,35)$. Published data did not permit a clear distinction between a monotonous decrease of the cell velocity with increasing adhesion strength beyond the velocity maximum and saturating effects of adhesion on the velocity. We have chosen a sufficiently small step size in coating densities and a sufficiently large number of measurements per data point to clearly identify saturation.

We also analyzed the velocity changes at transitions between different Fibronectin densities with regard to the biphasic relation at the single-cell level (Fig. 2C and Movie S3). Cells move from fields with coating Fibronectin density, Fibronectin ${ }_{i}$,

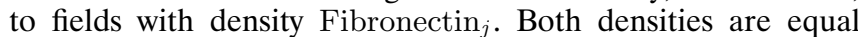
on the diagonal (Fibronectin ${ }_{i}=$ Fibronectin $_{j}$ ) of Fig. $2 C$. We have transitions from low to high density above the diagonal. Fig. $2 C$ presents increases (green), decreases (red), and no change (white) of the median relative velocity of single cells during transitions. Evidently, the velocities on both densities are approximately equal on the diagonal with Fibronectin $_{i}=$ Fibronectin $_{j}$ (full line in Fig. $2 C$ ). Interestingly, there are distinct pairs of Fibronectin concentrations around the density with maximal velocity Fibronectin max $_{\text {, where, despite }}$ a sharp step in Fibronectin density, the cell velocities do not change. This consequence of the biphasic relation manifests itself as a second weakly white diagonal line Fibronectin $_{j}=$ Fibronectin $_{\max }-$ Fibronectin $_{i}$ (dashed line in Fig. 2C). This 
A

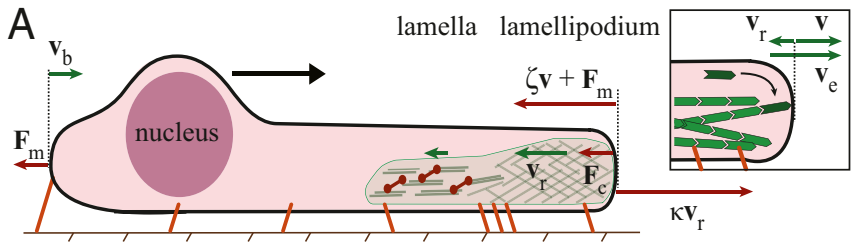

B

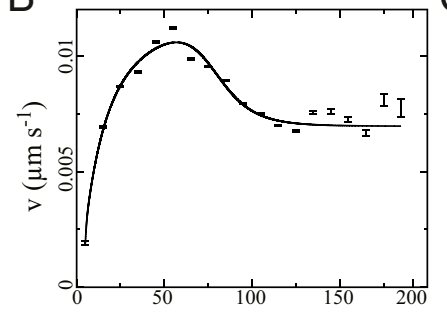

Fibronectin $\left(\mathrm{ng} \mathrm{cm}^{-2}\right)$

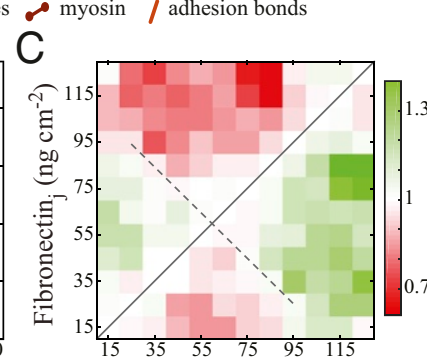

Fibronectin $_{\mathrm{i}}\left(\mathrm{ng} \mathrm{cm}^{-2}\right)$

Fig. 2. The adhesion-velocity relation. (A) Sketch of a cell with velocities of the leading edge $v$, the retrograde flow $v_{r}$, the network-extension rate $v_{e}$ the back velocity $v_{b}$, and the related elements of the force balance of steady motion $\zeta v$ and $\kappa v_{r} . F_{c}$ and $F_{m}$ are forces acting on the cell membrane. $F_{m}$ could be the force from an obstacle to motion or pulling the rear, for example. (B) Measured adhesion-velocity relation of MDA-MB-231 cells (symbols). The full line shows the fit to Eqs. $\mathbf{4}$ and $\mathbf{5}$ with the parameters listed in Table 1; bars show SEM. Each data point contains, on average, 1,000 cell tracks, but with decreasing numbers for very large and very small Fibronectin densities (SI Appendix, Table S6). (C) Relative velocities for all combinations of different Fibronectin densities. Distribution of relative velocities before and after transitions from Fibronectin ${ }_{i}$ to Fibronectin F $_{j}$ have been measured for each pair of densities. The color coding compares the relation between the medians: green for $v_{j}>v_{i}$, white for $v_{j}=v_{i}$, and red for $v_{j}<v_{i}$. Lines indicate combinations of Fibronectin densities where the velocities on both of them are equal.

line separates two regimes: below the line Fibronectin $_{j}=$ Fibronectin $_{\text {max }}-$ Fibronectin $_{i}$, we observe the rising phase of the adhesion-velocity relation with increasing velocities. Above this line, we enter the falling phase of this relation and see decreasing velocities during the transition from Fibronectin ${ }_{i}$ to Fibronectin $_{j}$. Below the diagonal Fibronectin ${ }_{i}=$ Fibronectin $_{j}$ we see transitions from high to low density.

Force Balance at the Leading Edge, Force-Dependent Polymerization, and Cooperative Adhesion Determine the Adhesion-Velocity Relation. In this section, we formulate a force balance at the leading edge in order to describe cell motion. Forces act on the cell across the whole area of contact with the substrate. They are coupled across the whole cell to the leading edge by tension (36, 48-51) or by the F-actin network for those parts of the network which are mechanically continuous with the lamellipodium (52). On that basis, we can place our force balance at the leading edge without neglecting forces acting on different parts of the cell, including the lamella.

We focus on the steady motion, when average front and rear velocity of the cell are equal on areas with homogeneous Fibronectin density in this section. Fig. $2 A$ shows the forces acting at the leading edge of the cell. Each individual filament pushes with its force $f$, which increases the activation energy for the polymerization reaction, since the addition of length to the filament has to work against $f$. That entails the Arrhenius factor $e^{-\frac{f d \cos \theta}{k_{B} T}}$ in the polymerization rate with $\theta$ denoting the tilt angle of the filament, $k_{B}$ the Boltzmann constant, and $T$ the temperature (53-55). We approximate the average single-filament force by the total force per leading-edge contour length $F$ divided

by the number of filaments per leading-edge contour length $N$. Since the extension velocity of the network is mainly determined by the polymerization rate, it obeys

$$
v_{e}=V_{e}^{0} e^{-\frac{g d}{k_{B} T} \frac{F}{N}}-k^{-},
$$

with the geometric factor $g$ resulting from averaging over the tiltangle distribution, the force-free extension velocity $V_{e}^{0}$, and the barbed-end depolymerization rate $k^{-}$. The most important contribution of the force dependency of the polymerization rate is a scale of single-filament force given by $a=g d / k_{B} T$, on the basis of which we can evaluate intracellular forces below.

The extension velocity $v_{e}$ is the vectorial difference of the protrusion velocity $v$ and retrograde-flow velocity $v_{r}$ (Fig. 2A). Their absolute values obey

$$
v_{e}=v+v_{r} .
$$

We formulate the force balance at the leading edge in terms of friction and drag forces proportional to velocities in the highly viscous environment of moving cells (low-Reynolds-number regime) and velocity-independent forces. The force at the leading edge required to drive retrograde flow is $\kappa v_{r}$, with the retrograde-flow friction coefficient $\kappa$ accounting for viscous resistance and friction between the F-actin network and intracellular structures. The force required to move the leading-edge membrane is $\zeta v$, and $\zeta$ comprises drag resistance of membrane motion, but also the detachment of adhesion bonds at the rear of the cell. Since the force $F$ pushing the leading-edge membrane also drives retrograde flow $(16,52)$ (SI Appendix, section S5), the force balance at the leading edge is

$$
F=\kappa v_{r}-F_{c}=\zeta v+F_{m} .
$$

$F_{c}$ is a force acting on the network at the leading edge like, for example, from contraction by myosin. Contractile forces are positive with our choice of sign in Eq. 3. $F_{m}$ is a velocity-independent force acting on the leading-edge membrane, e.g., myosin activity pulling the rear or an obstacle to cell motion.

The basic thermodynamic relation Eq. 1 and the force balance Eq. 3 determine the cell velocity $v$ uniquely. The solution uses the Lambert function $W_{0}(56)$ :

$$
\begin{aligned}
v= & \frac{N}{a \zeta} W_{0}\left(\frac{a V_{e}^{0} \kappa \zeta}{N(\zeta+\kappa)} e^{\frac{a}{N(\zeta+\kappa)}\left(\zeta\left(F_{c}+\kappa k^{-}\right)-\kappa F_{m}\right)}\right) \\
& -\frac{F_{c}+F_{m}+\kappa k^{-}}{\zeta+\kappa} .
\end{aligned}
$$

Eq. 4 relates the cell velocity directly or indirectly to many experimental parameters. In particular, both the retrograde-flow friction coefficient $\kappa$ as well as the drag coefficient $\zeta$ depend on adhesion. Adhesion determines the density of stationary structures inside the cell and, thus, controls coupling of retrograde actin flow to the substrate. It also determines the force required to pull the rear of the cell off the adhesion bonds and, thus, affects $\zeta$.

Binding of a ligand to integrin is one of the most important ways how cells sense their environment. The binding event is input to a complex signaling network $(27,34,57,58)$. The signaling state of related pathways-e.g., Rho signaling to stress fibers $(27,57,59-61)$ or Rac signaling to FAs $(25,59,62-64)$ and other systems (65-68) - and force-, flow-, and myosin-mediated feedbacks affect the density of adhesion structures $(8,20,23,31$, $60,69-73)$, interact with integrin signaling, and thus shape the relations between $\kappa, \zeta$, and the ligand density $B$. In the different experimental settings, $B$ represents the Fibronectin density, 
Table 1. Parameter values resulting from the fits of Eqs. 4 and 5 to experimental data shown in Figs. $2 B$ and 3

\begin{tabular}{|c|c|c|c|c|c|}
\hline Parameter & MDA-MB-231 & $\mathrm{CHO}$ & Keratocytes & PtK1 & Units \\
\hline \multicolumn{6}{|l|}{ F-actin network extension Eq. 1} \\
\hline Force-free network-extension rate $V_{e}^{0}$ & 0.0156 & 0.057 & 0.197 & 0.030 & $\mu \mathrm{m} \cdot \mathrm{s}^{-1}$ \\
\hline Network depolymerization rate $k^{-}$ & 0.0027 & 0.010 & 0.0017 & 0.0016 & $\mu \mathrm{m} \cdot \mathrm{s}^{-1}$ \\
\hline \multicolumn{6}{|l|}{ Retrograde-flow friction coeff. $\kappa(B)$ Eq. 5} \\
\hline Hill coefficient $n_{\kappa}$ & 8.77 & 7.71 & 1.94 & 2.11 & \\
\hline Half-max. value $K_{\kappa}$ & 73.6 & 4.63 & 66.1 & 22.3 & $\mu \mathrm{g} \cdot \mathrm{mL}^{-1}, \mathrm{ng} \cdot \mathrm{cm}^{-2}$ \\
\hline Max. value $\kappa_{\max }$ & 70.1 & 13.8 & 6.12 & 2.60 & $\mathrm{nN} \cdot \mathrm{s} \cdot \mu \mathrm{m}^{-2}$ \\
\hline \multicolumn{6}{|l|}{ Membrane drag coefficient $\zeta(B)$ Eq. 5} \\
\hline Hill coefficient $n_{\zeta}$ & 7.59 & 7.53 & 1.36 & 1.53 & \\
\hline Half-max. value $K_{\zeta}$ & 85.7 & 5.33 & 218.6 & 382 & $\mu \mathrm{g} \cdot \mathrm{mL}^{-1}, \mathrm{ng} \cdot \mathrm{cm}^{-2}$ \\
\hline Max. value $\zeta_{\max }$ & 30.8 & 296.0 & 2.86 & 117 & $\mathrm{nN} \cdot \mathrm{s} \cdot \mu \mathrm{m}^{-2}$ \\
\hline \multicolumn{6}{|l|}{ Forces Eq.3 and SI Appendix, Eq. S8 } \\
\hline$F_{c}$ & 0 & 0 & 0 & 0 & $\mathrm{nN} \cdot \mu \mathrm{m}^{-1}$ \\
\hline$F_{m}$ & 0 & 0 & 0 & 0 & $\mathrm{nN} \cdot \mu \mathrm{m}^{-1}$ \\
\hline$\frac{F_{\max }^{\text {pol }}}{N}$ & 0.007 & 0.007 & 0.019 & 0.012 & $\mathrm{nN}$ \\
\hline
\end{tabular}

The values $g=0.375(79), g d / k_{B} T=248 \mathrm{nN}^{-1}$, and $N=248 \mu \mathrm{m}^{-1}(17,77)$ were used for all fits. SI Appendix, section S4 relates some parameter values to available published results. Coeff., coefficient; max., maximum.

Fibrinogen concentration, or Arg-Gly-Asp (RGD) functionalized poly-L-lysine-graft-PEG copolymer (PLL-PEG-RGD) density (see below). Catch bonds between integrin and Fibronectin (74) or actin and vinculin (75) will contribute to the positive feedback in this relation. We will explore the relations $\kappa(B)$ and $\zeta(B)$ by fitting the velocity Eq. 4 to experimental results. The complexity of adhesion-related pathways (see refs. 9, 10, 27, 34, 57, 58,66 , and 76 for reviews) entails most likely a more complicated relation between $\kappa, \zeta$, and ligand density than the type we will choose, but we cannot expect to learn all of the details of this relation from our experimental data and, therefore, have to choose a sufficiently simple ansatz.

According to the basic ideas on the biphasic adhesion-velocity relation, $\kappa(B)$ and $\zeta(B)$ should increase with $B$. The observation that velocities saturate for large Fibronectin densities in Fig. $2 B$ requires that $\kappa(B)$ and $\zeta(B)$ are also saturating functions. Therefore, we choose the ansatzes

$$
\kappa(B)=\frac{\kappa_{\max } B^{n_{\kappa}}}{B^{n_{\kappa}}+K_{\kappa}^{n_{\kappa}}}, \quad \zeta(B)=\frac{\zeta_{\max } B^{n_{\zeta}}}{B^{n_{\zeta}}+K_{\zeta}^{n_{\zeta}}}
$$

The fits provide most of the parameters of Eqs. 4 and 5, but the scaling properties of this equation do not allow for fitting a complete parameter value set. A factor multiplying either $\kappa(B)$ or $\zeta(B)$ like the parameter ratio $a / N$ can be scaled out of the equation and, therefore, cannot be determined. We have chosen the value $n=248 \mu^{-1}$, based on measured values in the range from 150 to $320(17,77,78)$. That value entails $a / N=1 \mu \mathrm{m} \cdot \mathrm{nN}^{-1}$.

Analysis of the Adhesion-Velocity Relation. We analyze the adhesion-velocity relation by fitting Eqs. $\mathbf{4}$ and $\mathbf{5}$ to the measured data in Fig. $2 B$ of the MDA-MB-231 dataset. The fit reproduces the relation, including the velocity maximum and the saturating behavior. The resulting parameters are listed in Table 1 . We also include data from literature for keratocytes (33), PtK1 cells (31), and CHO cells (7) into the analysis (Figs. 3 and 4 and Table 1). We calculate the force $F$, retrograde-flow velocity $v_{r}$, and network-extension rate $v_{e}$ with the parameters from the fits and Eqs. 2-5.

Keratocytes offer the best opportunity for relating our results to prior knowledge, since they are the best-studied cell type among our examples. The forces occurring in keratocyte migration on coating densities covered by the experiments are in the same range (Fig. 4) as the forces of the dynamic (14-16) and pre- dicted stationary force-velocity relation (SI Appendix, Fig. S4), and are in agreement with experimental and theoretical analyses of membrane tension in moving keratocytes (80). Keratocytes
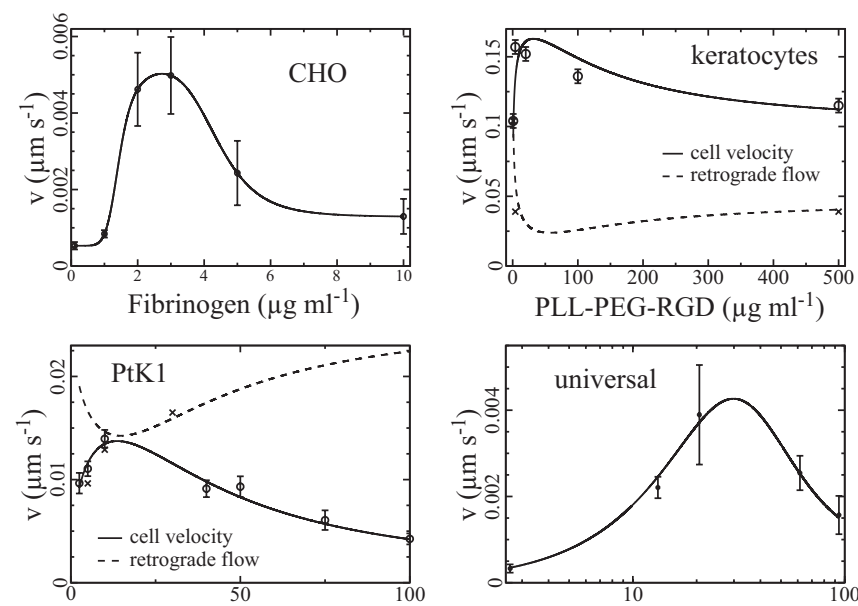

Fibronectin $\left(\mu \mathrm{g} \mathrm{ml}^{-1}\right)$

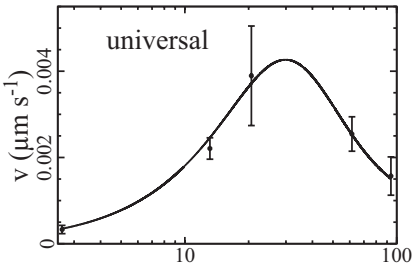

detachment force $(\mathrm{nN})$

Fig. 3. The dependency of the cell velocity on the substrate ligand den sity in terms of the concentration of Fibrinogen in the coating solution for $\mathrm{CHO}$ cells (data from the $\alpha_{i i b} \beta_{3}$ resting cells in ref. 7), the concentration of Fibronectin for PtK1 cells (data from ref. 31), the concentration of PLLPEG-RGD for keratocytes (data from ref. 33). The sets of experimental data (symbols) were fitted to Eqs. 4 and 5. The parameter value results are listed in Table 1. There are also retrograde-flow data $(x)$ available for keratocytes and PtK1 cells, which we included in the fit. The fit for PtK1 cells deviates in the data point of retrograde flow at $5 \mu \mathrm{g} \cdot \mathrm{mL}^{-1}$ from the experimental values. We discuss that deviation in SI Appendix, section S4A. Palecek et al. (7) could collapse several experimental sets to a single universal curve by relating the velocity to the detachment force, which is proportional to the adhesion bond density. We fit this universal relation data to Eqs. 4 and $\mathbf{5}$ in the universal graph (Lower Right). However, the fit is not unique with five data points only, and, therefore, we did not continue the analysis with this dataset (results in SI Appendix, Table S1). We found the introduction of (small) coating density-independent terms $\kappa_{0}=8 \times 10^{-5} \mathrm{nN} \cdot \mathrm{s} \cdot \mu \mathrm{m}^{-2}$ and $\zeta_{0}=7 \times 10^{-4} \mathrm{nN} \cdot \mathrm{s} \cdot \mu \mathrm{m}^{-2}$ in Eq. 5 to be necessary to obtain the inflection point the velocity dependency of the $\mathrm{CHO}$ cells exhibits left of the maximum. They may indicate the relevance of physical interactions between cell and substrate or coating-independent surface bonds. Bars indicate SEM for PtK1 cells and keratocytes and $95 \% \mathrm{Cl}$ for $\mathrm{CHO}$ cells and the universal dataset. 

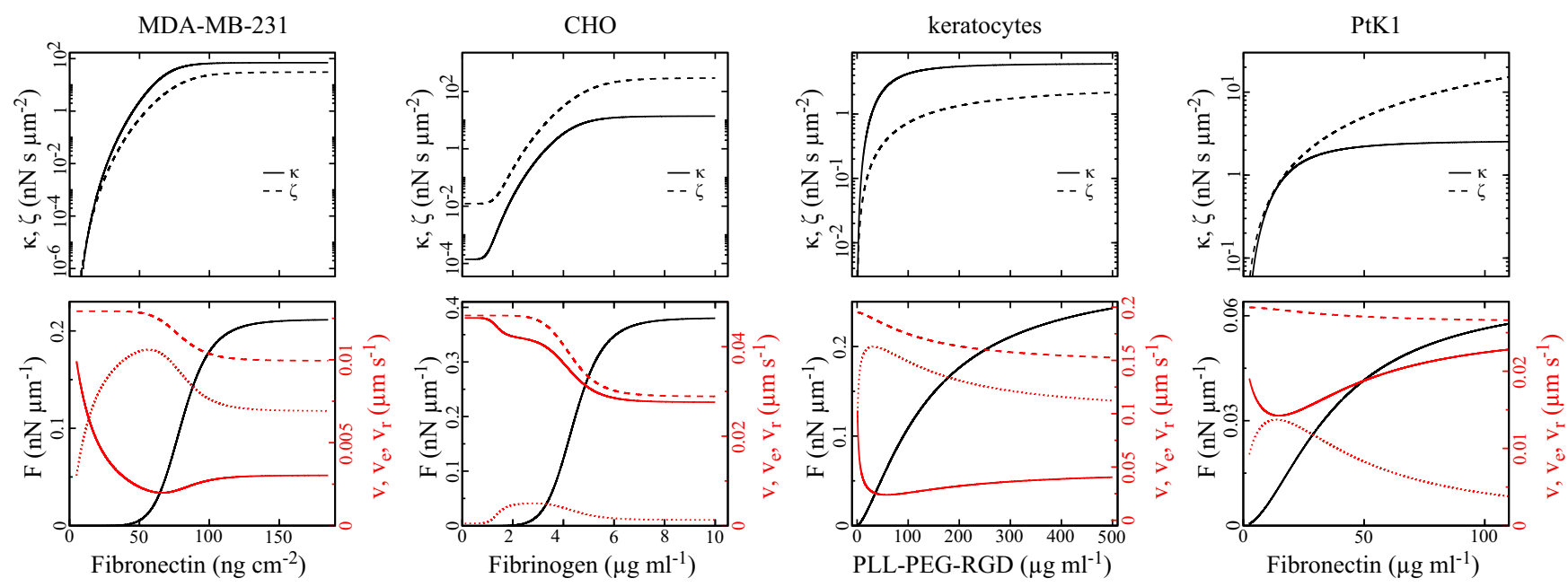

Fig. 4. Predictions of Eqs. 2-5 with the parameters for the different cell types listed in Table 1 resulting from the fits of Eqs. 4 and $\mathbf{5}$ to experimental data shown in Figs. $2 B$ and 3. (Upper) Retrograde-flow velocity friction coefficient $\kappa$ and membrane drag coefficient $\zeta$ (Eq. 5). (Lower) Network-extension rate $v_{\mathrm{e}}$ (red dashed line; Eq. 2), retrograde-flow velocity $v_{r}$ (red full line; Eq. 3), cell velocity $v$ (red dotted line; Eq. 4), and the force $F$ (black full line; Eq. 3) acting on the leading-edge membrane.

exhibit $\kappa \gg \zeta$ (Fig. 4) in agreement with observations by Anderson and Cross (81) and Möhl et al. (82) for fish and human epidermal keratinocytes, respectively (resp.), showing vanishing adhesion density toward the trailing edge of the cell. The maximum of the cell velocity is at intermediate $\kappa$ values, in agreement with experimental results on adhesion-site density for different Fibronectin-coating densities (31). SI Appendix, Table S3 provides additional information. The high quality of the fits to the experimental results in Figs. 2 and 3 and the quantitative agreement with prior knowledge on keratocytes strongly suggests that we included all relevant processes in the formulation of the velocity equation (Eq. 4).

Forces. Interestingly, fitting reveals that both velocity-independent forces vanish, $F_{m}=0$ and $F_{c}=0$, for all experiments but the universal set, and, there, $F_{m}=0$ and $F_{c}$ is tiny (SI Appendix, Table S1). Hence, velocity-independent forces do not contribute to the leading-edge force balance in a noticeable way (see also Discussion). This finding also entails $v / v_{r}=\kappa / \zeta$ (Eq. 3). Hence, we can measure the ratio $\kappa / \zeta$ by measuring $v / v_{r}$.

The force $F$ increases, and the network-extension rate $v_{e}$ decreases with increasing substrate-coating density for all four cell types in Fig. 4, in line with Eq. 1. If we see a substantial decrease of $v_{e}$ as with MDA-MB-231 and CHO cells, it starts at coating densities close to the velocity maximum. The extension velocity decreases typically by less than $30 \%$, but this amount is the larger part of the cell-velocity decrease beyond the maximum in MDA-MB-231 cells, keratocytes, and CHO cells.

PtK1 and $\mathrm{CHO}$ cells with $\zeta \geq \kappa$ turn a large fraction of the network-extension rate into retrograde flow. This correlates to the force regime in which these cells operate. We can assess the force regime by inspecting the stall forces (83), i.e., the strength of a force $F_{m}$ required to stall leading-edge motion:

$$
F_{m}^{\text {stall }}=\frac{N}{a} W_{0}\left(\frac{a V_{e}^{0} \kappa}{N} e^{\frac{a}{N}\left(F_{c}+k^{-} \kappa\right)}\right)-\kappa k^{-}-F_{c} .
$$

The force $F$ acting on the leading-edge membrane is very close to $F_{m}^{\text {stall }}$ if $\zeta /(\kappa+\zeta) \approx 1$ or $\kappa \ll \zeta$ holds (see also Eq. 4), i.e., if resistance to motion is large. Indeed, the force in PtK1 and CHO cells is close to $F_{m}^{\text {stall }}$ (SI Appendix, Fig. S5), and $\kappa$ is much smaller than $\zeta$ (Fig. 4). The CHO cells exhibit even $F \approx F_{m}^{\text {stall }}(S I$
Appendix, Fig. S5) and, consequently, move with tiny cell velocities. Forces in all four cell types are one order of magnitude below the maximally possible force $F_{\max }^{\text {pol }}$ (SI Appendix, Eq. S8), where polymerization stops, and which is reached with very large $\kappa$ only (SI Appendix, Figs. S5 and S6).

We find both stronger effects of adhesion on protrusion force at the front $\kappa \geq \zeta$ (MDA-MB-231 and keratocytes) and stronger effects of adhesion on resisting force $\zeta \geq \kappa$ (CHO cells and PtK1 cells). Pushing $\left(\kappa v_{r}\right)$ and resisting $(\zeta v)$ forces are not only determined by $\kappa$ and $\zeta$, but also by retrograde flow and cell velocity. Pushing may be strong due to fast retrograde flow, despite small $\kappa$ (PtK1 cells), and resistance may be small due to slow cell velocity, despite large $\zeta$ (CHO cells). Hence, motion is possible in both cases, when adhesion effects on pushing forces (large $\kappa$ ) or on resisting forces (large $\zeta$ ) dominate.

The relations between the ligand density $B$ and the friction coefficient $(\kappa(B))$ and drag coefficient $(\zeta(B))$, respectively, hold information on the signaling pathway from integrin to adhesion structures.

The values of $\kappa$ and $\zeta$ increase with increasing Fibronectin, Fibrinogen, or PLL-PEG-RGD density, in accordance with the idea that higher ligand density causes more adhesion structures, entailing larger friction and drag. We illustrate adhesion structures by staining of Paxillin, an adhesion-site protein, and F-actin in Fig. $5 A$ and SI Appendix, Fig. S13. The MDA-MB-231 cell in Fig. $5 A$ exhibits high adhesion-site density a few micrometers behind the leading edge, where also stress fibers are anchored (Factin image). F-actin density is the highest in the lamellipodium. SI Appendix, Fig. S13 shows larger regions with high adhesionsite density on high Fibronectin (SI Appendix, Fig. S13A) than on low Fibronectin (SI Appendix, Fig. S13B) and, thus, corroborates the relation between ligand density and adhesion structures.

Our fitting results show rather strong positive feedback in the pathway from integrin to adhesion structures in MDA-MB-231 and CHO cells (values of $n_{\kappa} \gg 1$ and $n_{\zeta} \gg 1$; Table 1 ). These cells exhibit a fast rise of the cell velocity by about a factor of six at low coating density (Figs. 2 and 3 ) and a steep rise of the force $F$ (Fig. 4). This likely reflects the feedbacks on adhesion strengthening mediated by myosin $(31,33,72,84)$ and other pathway components (8), by F-actin flow (20), the effect of force on adhesion strength, and possibly catch bonds $(9,60,69,70$, 

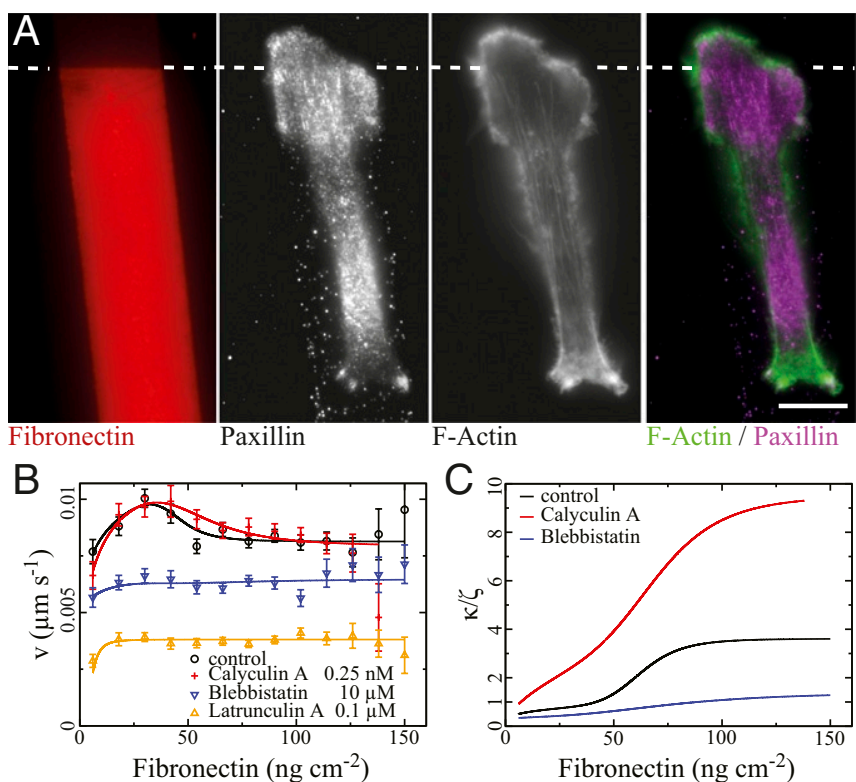

Fig. 5. F-actin, adhesion sites, and effects of inhibitors in MDA-MB-231 cells. (A) Fixed cell on a Fibronectin step (moving up, 8 to $40 \mathrm{ng} \cdot \mathrm{cm}^{-2}$ ). Images of labeled Fibronectin and Phalloidin-stained F-actin are acquired with epifluorescence, and antibody-stained Paxillin with total internal reflection fluorescence microscopy. (Scale bar: $10 \mu \mathrm{m}$.) $(B)$ Velocity- adhesion relation of MDA-MB-231 H2B mCherry control cells and cells treated with $10 \mu \mathrm{M}( \pm$ )-Blebbistatin, $0.1 \mu \mathrm{M}$ Latrunculin $\mathrm{A}$, or $0.25 \mathrm{nM}$ Calyculin A. (C) Ratios of friction coefficients $\kappa$ and $\zeta$ resulting from the fits in $B$. A different batch of labeled Fibronectin was used compared to Fig. 2. (For a discussion of absolute Fibronectin densities, see SI Appendix, section S1B.)

73-75, 85). PtK1 cells and keratocytes show values of $n_{\kappa}$ and $n_{\zeta}$ between 1.4 and 2.1; the initial rise of velocities is by a factor of about 1.5 only; and the force rises much slower and does not even saturate within the investigated range of substrate coatingdensity values (Figs. 3 and 4). The small values of $n_{\kappa}$ and $n_{\zeta}$ in keratocytes might be related to the spatial distribution and direction of myosin-related stress in these cells. The wings and lateral extensions show the strongest response to ligand-density increase (33), and stress orthogonal to the direction of motion is about five times stronger than in the direction of motion (22, $86)$. Hence, a large part of the response may not contribute to propulsion.

We learn more about the positive feedback by applying Blebbistatin, Calyculin A, or Latrunculin A. Blebbistatin inhibits myosin II activity. Its application $(10 \mu \mathrm{M})$ reduces the cell velocity by about $40 \%$ (Fig. $5 C$ ). The maximum of the velocity in dependence on Fibronectin density vanished. The curve is much closer to a monotonously increasing relation than a biphasic relation. The values of both $n_{\kappa}$ and $n_{\zeta}$ are substantially reduced to about 2.8 in comparison to about 6.5 of the control data and about 4.5 of the Calyculin A data (SI Appendix, Table S2), indicating that myosin suppression removes an essential element of the positive feedback from Fibronectin to adhesion structures. The ratio of the coefficients $\kappa$-entailing protrusive forcesand $\zeta$-entailing resisting forces-illustrates the action of the drugs on the positive feedback. The ratio grows with increasing Fibronectin density in control cells. Blebbistatin abolishes large $\kappa$ to $\zeta$ ratios.

Calyculin A amplifies myosin II activity. Its application (0.25 $\mathrm{nM}$ ) caused a broader maximum of the velocity (Fig. $5 C$ ). This shape of the relation entails a decrease of $n_{\kappa}$ and $n_{\zeta}$ from control values of about 6.5 to 4.7 and 4.1, resp., since the slope from the maximum toward the saturation value is less steep (Fig. $5 C$ and
SI Appendix, Table S2). Application of Calyculin A entails the largest ratio $\kappa / \zeta$, indicating a strong increase of the density of adhesion structures in areas with retrograde flow.

Latrunculin A inhibits F-actin polymerization. Its application $(0.1 \mu \mathrm{M})$ entails much smaller velocity than control and also a monotonously increasing relation. In line with the effect of the drug, the value of the parameter quantifying the force-free polymerization $V_{e}^{0}$ was determined as about one-fifth of the control value by the fit (SI Appendix, Table S2). Latrunculin A also substantially increased the half-maximum values $K_{\kappa}$ and $K_{\zeta}$, rendering precise fit results for $n_{\kappa}, \kappa_{\max }, n_{\zeta}$, and $\zeta_{\max }$ difficult (SI Appendix). In the end, the large values of $K_{\kappa}$ and $K_{\zeta}$ also abolish positive feedback and entail a monotonous dependency of $v$ on Fibronectin.

\section{Fibronectin Density Steps Reveal Spring-Like Interaction of Front and} Rear. We have considered steady motion of cells on homogeneous Fibronectin density so far. Characterizing the mechanical properties of the front-rear interaction requires perturbations of the motion on homogeneous coating, as given by the transitions between different Fibronectin densities (Fig. $6 \mathrm{~A}$ and $B$ ). Cell front and rear see different ligand densities during transitions between density fields (Fig. $6 A$ ). The velocity of the cell in Fig. $6 B$ increases when its front part enters a region with high adhesion density and slightly decreases when its rear part also reaches the high-Fibronectin region (Fig. $6 A$ and $B$ ). When the cell enters a region with low Fibronectin density, it shows the opposite behavior with decreased velocity during the transition and an increase when the transition is completed (Fig. $6 \mathrm{~A}$ and $B)$. This directly confirms the idea that strong adhesion at the front supports motion, and strong adhesion at the back resists motion.

Kymographs of another set of measurements with 20-s time resolution show that the front is moving rather smoothly and changes its velocity at the Fibronectin density steps (Fig. $6 B$ and Movie S4). The rear motion exhibits larger fluctuations and can even form a transient protrusion extending backward. We consider the front velocity $v$ as the cell velocity and, additionally, introduce the velocity of the back $v_{b}$. Although $v_{b}$ fluctuates, it is, on average, equal to $v$ during steady-state motion.

We need to look closer at the force-resisting motion to understand the differential behavior of $v$ and $v_{b}$. We will see below that the cell length $L$ is mainly determined by the Fibronectin density at the rear of the cell, while the velocity is affected by both front and rear density. Based on that observation, we split the resisting force up into the drag force required to pull the front part and cell body on the substrate, $\zeta_{c} v$, and the force required to pull the cell rear, $\zeta_{b} v_{b}$ (Fig. $6 C$ ). The force balance also includes the length dynamics $\dot{L}$ now, and is given by (taking $F_{c}=F_{m}=0$ into account)

$$
\zeta v-\zeta_{b} \dot{L}=\zeta_{c} v+\zeta_{b} v_{b}=\kappa v_{r} .
$$

It takes a cell about 50 min to travel across a Fibronectin step. That is sufficiently slow to reach a stationary velocity, even during a transition when front and back move on different coating densities, but with the same velocity, $v=v_{b}$. Then, $\dot{L}=0$; $\kappa v_{r}=\left(\zeta_{c}+\zeta_{b}\right) v$ and $\zeta=\zeta_{c}+\zeta_{b}$ hold.

We start our experimental investigations on the nature of the front-rear coupling with the question for an intrinsic Fibronectin-related cell length. If it exists, the cell length should mainly be set by the Fibronectin density $B$, and not by the history of the cell motion. We measure the length of a cell on one density and the length after transitions to the other density and back. The distribution of the ratio of these two lengths peaks around one (Fig. $6 D$ and $E$ ); hence, cells have an intrinsic Fibronectin-density-dependent length $L_{0}(B)$. This motivates our choice for the force resisting cell stretching $F_{S}$ to be an 

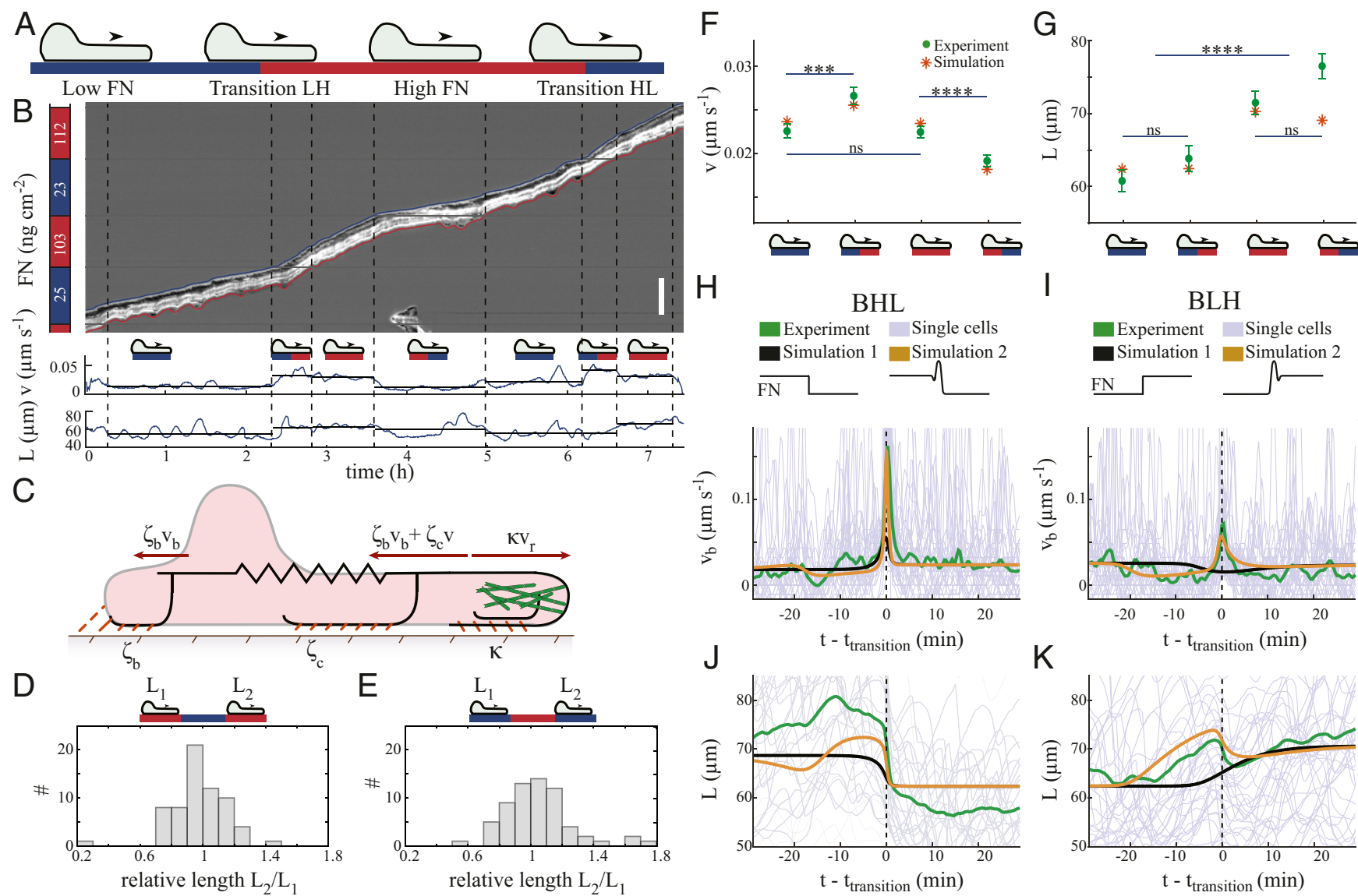

Fig. 6. Perturbation of steady motion at Fibronectin density steps. (A) We distinguish phases where cells are completely on one density segment, and transition phases where cell front and back are on different densities. FN, Fibronectin. (B) Kymograph of a cell running through areas of different Fibronectin densities (given on the left). We trace front (blue) and back (red) and provide the time course of front velocity (v) and cell length (L). Mean velocities and mean lengths are indicated by horizontal lines for each phase. (Scale bar: $100 \mu \mathrm{m}$.) $(C)$ Sketch of the cell model with spring-like front-rear interaction force. ( $D$ and $E$ ) The ratio of the mean lengths of individual cells after to before crossing a density region. $(F)$ Front velocity averaged over individual phases measured from 99 kymographs set in relation to simulation results. Error bars show SEM. (G) Length averaged for individual phases from the same kymographs as in $E$ and compared to simulation results. Results of two-sided $t$ test are indicated. ${ }^{* * *} P<0.001 ;{ }^{* * *} P<0.0001$. ns, not significant. ( $H$ and I) Back velocity during a transition of the back (B) from high $(\mathrm{H})$ to low $(\mathrm{L})$ Fibronectin $(\mathrm{BHL})$ and low to high (BLH). ( $J$ and $K)$ Cell length for BHL and BLH transitions. $(H-K)$ Thin gray lines are individual measured trajectories; the green line shows their average values. The black lines show the simulated time courses for a simple step profile of the Fibronectin density. Simulation results with the detailed Fibronectin profile with additional bumps as in Fig. 1 are shown by the yellow lines.

elastic one $F_{S}=E\left(L-L_{0}\right)$. This elastic force-length relation is a phenomenological description. We cannot conclude from our data how cells realize this spring-like behavior. It might be the one-dimensional analogon to a preferred shape in two dimensions $(33,87,88)$ or might be realized by the fast front-rear interaction reported by Maiuri et al. (89) or by other means.

The elastic force $F_{S}$ mediates front-rear interaction and, thus, the dependency of the cell velocity on the coating densities at both ends. The force $\kappa v_{r}$ pushing at the front drags the cell $\left(\zeta_{c} v\right)$ and stretches it by the force pulling on the rear $\zeta_{b} v_{b}=E\left(L-L_{0}\right)$ (Fig. $6 C$ ). Fig. $6 F$ summarizes this effect for the four different density combinations by averages across all measured data points in the different regimes. The adhesion-velocity relation Eq. 4 accounts not only for homogeneous Fibronectin density, but also for the situations with differential densities at front and back (see also SI Appendix, Eqs. S13-S16). We use it to fix the $\zeta$ values for these situations based on the measured velocities. Our analysis shows $\zeta$ to be small when the front of the cell is on low density and large when the front is on high density (SI Appendix, section S7, Figs. S9 and S10, and Table S5). We can also quantify $\zeta_{b}$ on the basis of the transitions between densities. The rear part $\zeta_{b} v$ of the force resisting motion stays small across the whole ligand- density range. Hence, if we see substantial resistance to motion, most of it is offered by the front part resisting force $\zeta_{c} v$ (Eq. 7; SI Appendix, Eqs. S17-S20).

The behavior of the cell length $L$ reflects the contributions of the cell parts to resistance. $L$ is the shortest when the rear of the cell is on low Fibronectin, and the force $\zeta_{b} v_{b}$ stretching the spring is small. It is the longest when the rear is on high Fibronectin, and stretching forces are larger (Fig. 6G; SI Appendix, Figs. S9 and S10), in agreement with results of Hennig et al. (90).

The biphasic character of the adhesion-velocity relation and cell variability entail a qualitative difference between two groups of cells with respect to the velocity behavior. Some cells moving across the density regions in a given experiment are in the rising phase of the adhesion-velocity relation and exhibit a smaller velocity $v$ on low Fibronectin than on high Fibronectin $\left(v_{L L}<v_{H H}\right)$, and some are in the falling phase and show $v_{L L}>$ $v_{H H}$ (see also SI Appendix, Fig. S12). Cells in the phase $v_{L L}<$ $v_{H H}$ of the adhesion-velocity relation show the higher velocities when the cell front is on high density, since increasing Fibronectin density also increases $\kappa$ and, therefore, the force generated by the front region (SI Appendix, Fig. S9). With cells in phase $v_{L L}>v_{H H}, \kappa$ is close to saturation on both high and low 
Fibronectin density (SI Appendix, Fig. S10). Increasing adhesion at the front increases force generation not via $\kappa$ in this phase, but by increasing retrograde flow on the expense of the cell velocity $v$. Therefore, velocities are the highest when resistance to motion is the lowest, i.e., when the cell rear is on low density. These observations nicely illustrate that the biphasic character of the adhesion-velocity relation results from competing forces.

The dynamic behavior during transitions between density regions shows qualitative differences of front versus back of the cell. The mean velocity of the front adapts quickly to the values set by the adhesion-velocity relation (SI Appendix, Fig. S11). During transitions of the front, the back velocity does not change dramatically, but follows the trend of the front velocity ( $S I$ Appendix, Fig. S11). In contrast, $v_{b}$ exhibits a pronounced peak when the cell back-traverses from high to low (BHL transition; Fig. $6 H$ ). This peak is reproduced by simulations (Fig. $6 H$ ). Surprisingly, we also find a peak of the back velocity during a transition of the back from low to high density (BLH), but smaller than for BHL transitions. If we use a simple step for the Fibronectin profile at the transition points, the BLH simulation does not show a peak in difference to the experiments (Fig. 6I). It turned out that details of the profile are relevant for this transition (Fig. 1A). The profile exhibits small density maxima bordering the high-density range and neighboring small minima next to them toward the interior of this range. When implementing this detailed Fibronectin profile, the results of simulations exhibit a positive peak of back velocity at the BLH transition, which is consistent with experimental data (Fig. 6 $H$ and $I$ ).

During BHL transitions, the average length of the cell shows a sudden drop (Fig. $6 J$ ), which corresponds to the peak of $v_{b}$. Additionally, we find an increase of the cell length prior to the transition. Simulations capture this behavior, if we use the detailed Fibronectin-density profile. Only the absolute changes of the cell length are slightly underestimated by the model. The length during BLH transitions increases before the transition, drops slightly during the transition, and subsequently increases again. Maybe the most remarkable result of the analysis of velocity and cell length during transitions is that also small Fibronectin-density variations, like the little bumps on the boundary of the density ranges, affect migration dynamics.

We quantify the elastic modulus $E$ of the force $F_{S}=E(L-$ $\left.L_{0}\right)$ by an analysis of the trailing-edge dynamics. It provides $E / \zeta_{b} \approx 0.011 \mathrm{~s}^{-1}$ (SI Appendix, Fig. S8). With the estimate for $\zeta_{b}$, we obtain $E=0.001$ to $0.0015 \mathrm{nN} \cdot \mu \mathrm{m}^{-2}$ (SI Appendix, Table S5). Considering $L_{0} \approx 50 \mu \mathrm{m}$ and an average cell height of $\approx 0.5 \mu \mathrm{m}$, we find a mean Young modulus of the cell of $\approx 0.10$ to $0.15 \mathrm{kPa}$ (SI Appendix, Eq. S1), which is in the range of Young moduli of adherent cells measured experimentally, $\approx 0.25 \mathrm{kPa}(91)$. We could reproduce the measured stationary and dynamic length behavior only by choosing a length-dependent force between front and rear. The time scale during which this force acts is in the range of a few minutes, which is longer than the visco-elastic relaxation time of cells (92). Therefore, a visco-elastic front-rear interaction force would not have been applicable. This confirms our choice of an elastic force for cell stretching in addition to the intrinsic length.

\section{Discussion}

We fabricated patterned Fibronectin lanes with well-defined steps in coating density by two-step microcontact printing and used them to study how adhesion acts on the balance of forces promoting and resisting motion, how forces are transmitted between cell front and rear, and how cells adapt their migration behavior to varying surface adhesiveness.

In the first part of the study, we used the biphasic adhesionvelocity relation to investigate force-generation mechanisms. In particular, our results from the analysis of cell transitions between different Fibronectin densities confirm the idea of adhesion-promoted pushing forces and adhesion-promoted forces resisting motion, which has been established in a variety of studies $(6-10,24,31,35,36)$. We are interested in the mechanism exploiting these forces for velocity control. The forces related directly to adhesion are traction forces, which form a dipole of opposing forces acting on the substrate $(93,94)$. Spread or moving cells adhered to the substrate exert forces in the range of $100 \mathrm{nN}(95)$ or tension in the kilopascal range $(24,93,96-98)$.

The forces controlling velocities appear to be much smaller. Our results (Fig. 4), direct force measurements (14-16), and membrane-tension measurements $(80,99)$ show forces related to motion acting on the cell membrane in the range of $0.1 \mathrm{nN} \cdot \mu \mathrm{m}^{-1}$. Ridley et al. state (59): "It is striking that the tractional forces measured in many studies far exceed what should be needed for cell translocation." If traction forces would directly control cell velocity, they would need to accomplish the difficult task of stabilizing a $0.1-\mathrm{nN}$ difference between two opposing forces of $100 \mathrm{nN}$. Additionally, we found that velocity-independent forces do not contribute to the adhesionvelocity relation $\left(F_{c}=0\right.$ and $F_{m}=0$; Eq. 3). The traction forces exerted by myosin II in stress fibers on their terminating adhesion sites are velocity-independent forces and balance. Altogether, these considerations suggest that gripping and sliding use distinct forces (see ref. 100 for a direct demonstration in fibroblasts).

With the mechanism our results support, it is not contractile tension that controls the velocity, but adhesion-controlled friction forces, which are three orders of magnitude smaller. The force generated by polymerization at the leading edge is transmitted to the substrate as a friction force in the lamellipodium and the lamella-lamellipodium transition zone. It is the force required to drive the retrograde flow of the F-actin network against friction in these regions. This idea is in agreement with the picture of a molecular clutch (19) and results on the dynamic force-velocity relation of lamellipodial protrusion (16). Retrograde flow in the lamella is driven by myosin $(20,25,31,101)$. Lamella flow also transmits traction stress to the substrate (25). If this stress is in the direction of retrograde flow, as in PtK1 cells, it also generates protrusive force (25).

Our results suggest that the protrusive forces generated in lamellipodium and lamella speed up cell motion in the rising phase of the adhesion-velocity relation, since the density of adhesion structures increases with the Fibronectin density, thus increasing friction and $\kappa$. Forces resisting motion are drag forces, possibly including a velocity-dependent dissociation rate of adhesion bonds (102). When these forces become larger with increasing adhesion strength, the velocity decreases again, due to the force dependency of the polymerization rate and/or faster retrograde flow on the expense of the protrusion velocity. That causes the falling phase of the adhesion-velocity relation. All parts of this mechanism work with velocity-related forces, which guarantees immediate feedback as a very simple means of coordinating front and rear velocity.

Application of Blebbistatin and Calyculin A has provided some insight into the role of myosin II in the mechanism of force generation. Traction stress under the lamella is substantially smaller than control upon Blebbistatin application (25). However, the leading edge still protrudes with suppressed myosin II activity (20); fibroblasts, $\mathrm{CHO}$ cells, and keratocytes still move $(100,103,104)$; spreading continues (101) or speeds up (105); and Gardel et al. (25) find actin-polymerization forces in the lamellipodium to be sufficient to generate traction and mediate leading-edge protrusion independent of myosin II activity in PtK1 cells. The cell velocity might even increase upon Blebbistatin application $(106,107)$. Suppression of polymerization by cytochalasin D stops retrograde flow in the lamellipodium and stops protrusion $(20,25,31,101)$, and the leading edge retracts 
toward larger adhesion structures $(20,101)$. Thus, motion with substantially reduced myosin II activity, retrograde flow, and traction transmission to the substrate in the lamella is possible, but motion without polymerization in the lamellipodium is not. At the same time, application of Blebbistatin decreases velocities and abolishes the biphasic character of the adhesion-velocity relation with MDA-MB-231 cells (Fig. 5C), PtK1 cells (31), and keratocytes (33). Amplifying myosin activity by Calyculin A entailed biphasic relations with higher velocity than control (Fig. 5C) (31, 33).

Partial understanding of this role of myosin II arises from an observation by Gupton and Waterman-Storer (31) on an effect of myosin II besides driving lamella flow by contraction. The density of adhesion structures decreased upon Blebbistatin application and increased upon Calyculin A application in PtK1 cells, i.e., myosin acts to a substantial part via its effect on adhesion structure density [also in other cell types $(106,107)]$. That means it shapes the dependency of $\kappa$ and $\zeta$ on the substrate ligand density $B$ (in terms of our model). The results by Gupton and Waterman-Storer (31) suggest myosin to be one of the factors causing cooperativity in that dependency, which renders $n_{\kappa}$ and $n_{\zeta}$ larger than one. Hence, Blebbistatin should weaken this cooperativity. Indeed, the Blebbistatin results with PtK1 cells and keratocytes are compatible with Hill coefficients close to one, like $n_{\kappa}=n_{\zeta}=1$ for PtK1 cells (31) and $n_{\kappa}=1.3$, $n_{\zeta}=1$ for keratocytes (33), and values of $\kappa$ and $\zeta$ smaller than control (SI Appendix, Fig. S14). We also found a substantial reduction of $n_{\kappa}$ and $n_{\zeta}$ values in MDA-MB-231 cells upon Blebbistatin application (Table 1; SI Appendix, Table S2).

We can conclude two aspects of the role of myosin II for the cell velocity from these considerations. Velocity-independent forces generated by myosin II-e.g., in stress fibers-balance and do not affect the cell velocity. Myosin II affects the density of adhesion structures and, thus, velocity-dependent friction forces via the friction coefficient and, thus, affects cell velocity. If that effect of myosin II tilts the balance toward $\kappa$, the velocity will decrease upon Blebbistatin application. It will increase if myosin tilts the balance toward $\zeta$. That might explain the differential effects of Blebbistatin application on cell velocity mentioned above.

Myosin also drives lamellar flow, causing friction forces via the flow velocity. However, we cannot individually quantify the contribution of this flow since we cannot separate it out of the total protrusive force. If lamella and lamellipodium are mechanically continuous, forces generated in the lamella are transmitted to the leading edge by affecting $\kappa$ and $\zeta(52)$. If they are discontinuous, the lamellar forces do not affect $\kappa$, but are transmitted to other cellular structures and thus affect $\zeta$, e.g., via membrane tension $(36,48-51)$. We cannot decide on the basis of our data whether lamella and lamellipodium are mechanically continuous, but, either way, they are taken into account by the leading-edge force balance.

The leading-edge force balance with our mechanism is $\kappa v_{r}=$ $\zeta v$, which entails two means to increase force at the front to compensate for stronger adhesion at the back: higher $\kappa$ by more front adhesion or faster retrograde flow by less protrusion. Our results indicate that cells use both of them. They (may) adapt adhesion $(19,31,33)$. Some cells (additionally) increase retrograde flow, when the effect of ligand density on $\kappa$ has saturated (Fig. 4; MDA-MB-231 cells, keratocytes, and PtK1 cells). The approximate conservation of network-extension rate supporting this mechanism has also been found in earlier studies $(18,108)$, even when the filament density changes by a factor of about two (79).

It has been argued before that a gradient of adhesion strength from strong at the front to weak at the back is required for motion $(37,38)$. Motion arises from contraction as a tug of war between strong and weak adhesion sites mediated by stress fibers in that picture, with the strong adhesions pulling off the weak ones and, thus, the cell forward. We find cells that move straight from one density range to the next one, even for large Fibronectin density steps, and both from low to high and from high to low densities. If front and back of the cell were in a tug of war, we would expect a limit in the difference of density allowing for high-low transitions where the net force is balanced, and cell migration would stop. However, we did not find such a limit within step heights up to $90 \mathrm{ng} \cdot \mathrm{cm}^{-2}$, which is essentially the dynamic range of the adhesion-velocity relation of MDAMB-231 cells (Fig. 2B). Additionally, we find that most of the resistance to motion is generated in the same region as pushing forces. Hence, we do not observe the spatial separation of the "teams" required for a tug of war. The forces pulling on the very rear of the cell are only a small contribution to the forces resisting motion. Nonetheless, they affect cell length when the rear is on high Fibronectin density.

Our results are in line with the ideas summarized by Munevar et al. (24) by the term frontal towing model, which identifies asymmetric force generation as the cause of motion based on traction stress measurements. The force balance $\kappa v_{r}=\zeta v$ shows this very clearly: Retrograde flow generated at the front entails protrusion with the velocity $v=v_{r} \kappa / \zeta$. If $\zeta$ is larger than $\kappa$, the velocity might be small, but the cell moves. Since we obtain motion even for small retrograde-flow velocities, the strength of force is not so important. Simply the fact that the force generated by polymerization is directed, points in a certain direction, is crucial here, i.e., the force's vectorial character-or polarization of the cell in biological terms.

Cells can move in heterogeneous environments with very adhesive and less adhesive regions in that way without getting stuck in the highly adhesive spots. If velocity-independent forces would contribute to the force balance, cell motion would start only beyond a critical retrograde flow $v_{r}=\left(F_{c}+F_{m}\right) / \kappa$. That critical value might not be reached by polymerization in highly adhesive substrate regions, and they might trap cells. Hence, relying only on velocity-dependent forces reduces the danger of trapping and is the more robust way of generating motion.

The ideas presented in this study supplement earlier studies on the pathways controlling F-actin density, myosin activation, and other feedbacks by a more mechanical view on the adhesion-velocity relation. The restriction to one-dimensional Fibronectin lanes presents itself as rewarding for the mechanistic study of this relation. Defined steps between different Fibronectin densities enable measurement of velocity and length adaptation and provide insights into the interaction between front and rear. The mathematical adhesion-velocity relation yields insights into the mechanism of velocity control by ligand density on the substrate. The relation holds for various cell types operating in different force regimes. It provides a quantitative mathematical framework for future studies on the relation of adhesion and the intracellular processes relevant for migration.

\section{Materials and Methods}

A detailed explanation of the materials and methods can be found in $\mathrm{SI}$ Appendix. It contains the protocols for micropatterning, Fibronectin-density measurements, cell culture, immunostaining, time-lapse microscopy, and image analysis.

Data Availability. All study data are included in the article and/or supporting information

ACKNOWLEDGMENTS We thank K. Rottner and J. Renkawitz for useful comments; T. Betz for providing cell lines; and C. Leu for the preparation of wafers. This work was supported by a grant from the German Science Foundation (DFG) through the Collaborative Research Center 1032 Project B01 (to J.O.R.); and DFG Gant FA 350/11-1 (to M.F.). 
1. K. Rottner, J. Faix, S. Bogdan, S. Linder, E. Kerkhoff, Actin assembly mechanisms at a glance. J. Cell Sci. 130, 3427-3435 (2017).

2. O. F. Omotade, S. L. Pollitt, J. Q. Zheng, Actin-based growth cone motility and guidance. Mol. Cell. Neurosci. 84, 4-10 (2017).

3. A. I. Bachir, A. R. Horwitz, W. J. Nelson, J. M. Bianchini, Actin-based adhesion modules mediate cell interactions with the extracellular matrix and neighboring cells. Cold Spring Harb. Perspect. Biol. 9, a023234 (2017).

4. M. Abercrombie, The Croonian lecture, 1978-the crawling movement of metazoan cells. Proc. Roy. Soc. Lond. B Biol. Sci. 207, 129-147 (1980).

5. M. P. Sheetz, D. P. Felsenfeld, C. G. Galbraith, Cell migration: Regulation of force on extracellular-matrix-integrin complexes. Trends Cell Biol. 8, 51-54 (1998).

6. A. Huttenlocher, M. H. Ginsberg, A. F. Horwitz, Modulation of cell migration by integrin-mediated cytoskeletal linkages and ligand-binding affinity. J. Cell Biol. 134, 1551-1562 (1996).

7. S. S. Palecek, J. C. Loftus, M. H. Ginsberg, D. A. Lauffenburger, A. F. Horwitz, Integrinligand binding properties govern cell migration speed through cell-substratum adhesiveness. Nature 385, 537-540 (1997).

8. M. Vicente-Manzanares, C. K. Choi, A. R. Horwitz, Integrins in cell migration-the actin connection. J. Cell Sci. 122, 199-206 (2009).

9. J. T. Parsons, A. R. Horwitz, M. A. Schwartz, Cell adhesion: Integrating cytoskeletal dynamics and cellular tension. Nat. Rev. Mol. Cell Biol. 11, 633-643 (2010).

10. K. Burridge, C. Guilluy, Focal adhesions, stress fibers and mechanical tension. Exp. Cell Res. 343, 14-20 (2016).

11. T. M. Svitkina, A. B. Verkhovsky, K. M. McQuade, G. G. Borisy, Analysis of the actinmyosin II system in fish epidermal keratocytes: Mechanism of cell body translocation. J. Cell Biol. 139, 397-415 (1997).

12. J. V. Small, T. Stradal, E. Vignal, K. Rottner, The lamellipodium: Where motility begins. Trends Cell Biol. 12, 112-120 (2002).

13. T. D. Pollard, The cytoskeleton, cellular motility and the reductionist agenda. Nature 422, 741-745 (2003).

14. M. Prass, K. Jacobson, A. Mogilner, M. Radmacher, Direct measurement of the lamellipodial protrusive force in a migrating cell. J. Cell Biol. 174, 767-772 (2006).

15. F. Heinemann, H. Doschke, M. Radmacher, Keratocyte lamellipodial protrusion is characterized by a concave force-velocity relation. Biophys. J. 100, 1420-1427 (2011).

16. J. Zimmermann et al., Actin filament elasticity and retrograde flow shape the forcevelocity relation of motile cells. Biophys. J. 102, 287-295 (2012)

17. F. Kage et al., FMNL formins boost lamellipodial force generation. Nat. Commun. 8 14832 (2017).

18. P. Vallotton, G. Danuser, S. Bohnet, J.-J. Meister, A. B. Verkhovsky, Tracking retrograde flow in keratocytes: News from the front. Mol. Biol. Cell 16, 1223-1231 (2005).

19. C. Jurado, J. R. Haserick, J. Lee, Slipping or gripping? Fluorescent speckle microscopy in fish keratocytes reveals two different mechanisms for generating a retrograde flow of actin. Mol. Biol. Cell 16, 507-518 (2005).

20. A. Y. Alexandrova et al., Comparative dynamics of retrograde actin flow and foca adhesions: Formation of nascent adhesions triggers transition from fast to slow flow. PloS One 3, e3234 (2008)

21. J. Renkawitz et al., Adaptive force transmission in amoeboid cell migration. Nat. Cell Biol. 11, 1438-1443 (2009).

22. M. F. Fournier, R. Sauser, D. Ambrosi, J.-J. Meister, A. B. Verkhovsky, Force transmission in migrating cells. J. Cell Biol. 188, 287-297 (2010).

23. D. Choquet, D. P. Felsenfeld, M. P. Sheetz, Extracellular matrix rigidity causes strengthening of integrin-cytoskeleton linkages. Cell 88, 39-48 (1997).

24. S. Munevar, Y. Wang, M. Dembo, Traction force microscopy of migrating normal and H-ras transformed 3T3 fibroblasts. Biophys. J. 80, 1744-1757 (2001).

25. M. L. Gardel et al., Traction stress in focal adhesions correlates biphasically with actin retrograde flow speed. J. Cell Biol. 183, 999-1005 (2008).

26. J. P. Heath, B. F. Holifield, Cell locomotion: New research tests old ideas on membrane and cytoskeletal flow. Cell Motil. 18, 245-257 (1991).

27. I. Kaverina, O. Krylyshkina, J. Victor Small, Regulation of substrate adhesion dynamics during cell motility. Int. J. Biochem. Cell Biol. 34, 746-761 (2002).

28. J. V. Small et al., Unraveling the structure of the lamellipodium. J. Microsc. 231, 479485 (2008).

29. M. Nemethova, S. Auinger, J. V. Small, Building the actin cytoskeleton: Filopodia contribute to the construction of contractile bundles in the lamella. J. Cell Biol. 180, 1233-1244 (2008).

30. C. K. Choi et al., Actin and $\alpha$-actinin orchestrate the assembly and maturation of nascent adhesions in a myosin ii motor-independent manner. Nat. Cell Biol. 10, 10391050 (2008).

31. S. L. Gupton, C. M. Waterman-Storer, Spatiotemporal feedback between actomyosin and focal-adhesion systems optimizes rapid cell migration. Cell 125, 1361-1374 (2006).

32. P. A. DiMilla, J. A. Stone, J. A. Quinn, S. M. Albelda, D. A. Lauffenburger, Maximal migration of human smooth muscle cells on fibronectin and type IV collagen occurs at an intermediate attachment strength. J. Cell Biol. 122, 729-737 (08 1993).

33. E. L. Barnhart, K.-C. Lee, K. Keren, A. Mogilner, J. A. Theriot, An adhesion-dependent switch between mechanisms that determine motile cell shape. PLoS Biol. 9, e1001059 (2011).

34. R. Zaidel-Bar, B. Geiger, The switchable integrin adhesome. J. Cell Sci. 123, 1385-1388 (2010).

35. B. Borm, R. P. Requardt, V. Herzog, G. Kirfel, Membrane ruffles in cell migration: Indicators of inefficient lamellipodia adhesion and compartments of actin filament reorganization. Exp. Cell Res. 302, 83-95 (2005).

36. J. Mueller et al., Load adaptation of lamellipodial actin networks. Cell 171 188-200e16 (2017)
37. P. A. DiMilla, K. Barbee, D. A. Lauffenburger, Mathematical model for the effects of adhesion and mechanics on cell migration speed. Biophys. J. 60, 15-37 (1991).

38. M. A. Gracheva, H. G. Othmer, A continuum model of motility in ameboid cells. Bull. Math. Biol. 66, 167-193 (2004).

39. R. S. Kane, S. Takayama, E. Ostuni, D. E. Ingber, G. M. Whitesides, Patterning protein and cells using soft lithography. Biomaterials 20, 2363-2376 (1999).

40. A. D. Doyle, F. W. Wang, K. Matsumoto, K. M. Yamada, One-dimensional topography underlies three-dimensional fibrillar cell migration. J. Cell Biol. 184, 481-490 (2009).

41. P. Maiuri et al., The first world cell race. Curr. Biol., 22(17):R673-R675, 2012.

42. C. Schreiber, et al., Ring-shaped microlanes and chemical barriers as a platform for probing single-cell migration. Sci. Rep. 6, 26858 (2016).

43. M. Théry et al., Anisotropy of cell adhesive microenvironment governs cell internal organization and orientation of polarity. Proc. Natl. Acad. Sci. U.S.A. 103, 19771 19776 (2006).

44. G. Mahmud et al., Directing cell motions on micropatterned ratchets. Nat. Phys. 5 606-612 (2009)

45. D. Caballero, J. Comelles, M. Piel, R. Voituriez, D. Riveline, Ratchetaxis: Long-range directed cell migration by local cues. Trends Cell Biol. 25, 815-827 (2015).

46. D. B. Brückner et al., Stochastic nonlinear dynamics of confined cell migration in twostate systems. Nat. Phys. 15, 595-601 (2019).

47. R. A. Desai, M. K. Khan, S. B. Gopal, C. S. Chen, Subcellular spatial segregation of integrin subtypes by patterned multicomponent surfaces. Integrative Biol. 3, 560-567 (2011)

48. A. R. Houk et al., Membrane tension maintains cell polarity by confining signals to the leading edge during neutrophil migration. Cell 148, 175-188 (2012).

49. E. L. Batchelder et al., Membrane tension regulates motility by controlling lamellipodium organization. Proc. Natl. Acad. Sci. U.S.A. 108, 11429-11434 (2011).

50. K. Keren, Membrane tension leads the way. Proc. Natl. Acad. Sci. U.S.A. 108, 1437914380 (2011)

51. N. C. Gauthier, T. A. Masters, M. P. Sheetz, Mechanical feedback between membrane tension and dynamics. Trends Cell Biol. 22, 527-535 (2012).

52. J. Zimmermann, M. Enculescu, M. Falcke, Leading edge-gel coupling in lamellipodium motion. Phys. Rev., 82, 051925 (2010).

53. A. Mogilner, G. Oster, Force generation by actin polymerization II: The elastic ratchet and tethered filaments. Biophys. J. 84, 1591-1605 (2003).

54. A. Gholami, J. Wilhelm, E. Frey, Entropic forces generated by grafted semiflexible polymers. Phys. Rev. 74, 041803 (2006)

55. M. J. Footer, J. W. J. Kerssemakers, J. A. Theriot, M. Dogterom, Direct measurement of force generation by actin filament polymerization using an optical trap. Proc. Natl. Acad. Sci. U.S.A. 104, 2181-2186 (2007).

56. I. S. Gradstein, J. M. Ryschik, Table of Integrals, Series, and Products (Academic Press, New York, NY, ed. 8, 2014)

57. E. Zamir, B. Geiger, Molecular complexity and dynamics of cell-matrix adhesions. $J$. Cell Sci. 114, 3583-3590 (2001).

58. X. Trepat, Z. Chen, K. Jacobson, Cell Migration (American Cancer Society, Atlanta, GA 2012), pp. 2369-2392.

59. A. J. Ridley et al., Cell migration: Integrating signals from front to back. Science 302 , 1704-1709 (2003)

60. M. Chrzanowska-Wodnicka, K. Burridge, Rho-stimulated contractility drives the formation of stress fibers and focal adhesions. J. Cell Biol. 133, 1403-1415 (1996).

61. M. Amano et al., Formation of actin stress fibers and focal adhesions enhanced by rho-kinase. Science 275, 1308-1311 (1997)

62. C. E. Turner, K. A. West, M. C. Brown, Paxillin-Arf gap signaling and the cytoskeleton Curr. Opin. Cell Biol. 13, 593-599 (2001).

63. D. J. Webb, FAK-Src signaling through paxillin, ERK and MLCK regulates adhesion disassembly. Nat. Cell Biol. 6, 154-161 (2004)

64. J. M. Bristow et al., The Rho-family GEF Asef2 activates Rac to modulate adhesion and actin dynamics and thereby regulate cell migration. J. Cell Sci. 122, $4535-4546$ (2009).

65. P. Hotulainen, P. Lappalainen, Stress fibers are generated by two distinct actin assembly mechanisms in motile cells. J. Cell Biol., 173, 383-394 (2006).

66. C. Ciobanasu, B. Faivre, C. Le Clainche, Actin dynamics associated with foca adhesions. Int. J. Cell Biol. 2012, 941292 (2012)

67. S. J. Franco et al., Calpain-mediated proteolysis of talin regulates adhesion dynamics. Nat. Cell Biol. 6, 977-983 (2004).

68. S. Stahnke et al., Loss of hem1 disrupts macrophage function and impacts on migration, phagocytosis and integrin-mediated adhesion. https://doi.org/10.1101/ 2020.03.24.005835 (25 March 2020).

69. T. Mitchison, M. Kirschner, Cytoskeletal dynamics and nerve growth. Neuron 1, 761 772 (1988).

70. D. G. Jay, The clutch hypothesis revisited: Ascribing the roles of actin-associated proteins in filopodial protrusion in the nerve growth cone. J. Neurobiol. 44, 114-125 (2000)

71. D. Riveline et al., Focal contacts as mechanosensors: Externally applied loca mechanical force induces growth of focal contacts by an mDia1-dependent and ROCK-independent mechanism. J. Cell Biol. 153, 1175-1186 (06 2001).

72. G. Giannone et al., Lamellipodial actin mechanically links myosin activity with adhesion-site formation. Cell 128, 561-575 (2007).

73. A. del Rio et al., Stretching single talin rod molecules activates vinculin binding. Science 323, 638-641 (2009).

74. F. Kong, A. J. García, A. P. Mould, M. J. Humphries, C. Zhu, Demonstration of catch bonds between an integrin and its ligand. J. Cell Biol. 185, 1275-1284 (2009).

75. D. L. Huang, N. A. Bax, C. D. Buckley, W. I. Weis, A. R. Dunn, Vinculin forms directionally asymmetric catch bond with F-actin. Science 357, 703-706 (2017)

76. T. M. E. Scales, M. Parsons, Spatial and temporal regulation of integrin signaling during cell migration. Curr. Opin. Cell Biol. 23, 562-568 (2011). 
77. V. C. Abraham, V. Krishnamurthi, D. Lansing Taylor, F. Lanni, The actin-based nanomachine at the leading edge of migrating cells. Biophys. J. 77, 1721-1732 (1999).

78. M. Vinzenz et al., Actin branching in the initiation and maintenance of lamellipodia. J. Cell Sci. 125, 2775-2785 (2012).

79. S. Dolati et al., On the relation between filament density, force generation, and protrusion rate in mesenchymal cell motility. Mol. Biol. Cell 29, 2674-2686 (2018).

80. Y. Schweitzer, A. D. Lieber, K. Keren, M. M. Kozlov, Theoretical analysis of membrane tension in moving cells. Biophys. J. 106, 84-92 (2014).

81. K. I. Anderson, R. Cross, Contact dynamics during keratocyte motility. Curr. Biol. 10, 253-260 (2000).

82. C. Möhl, N. Kirchgessner, C. Schäfer, B. Hoffmann, R. Merkel, Quantitative mapping of averaged focal adhesion dynamics in migrating cells by shape normalization. J. Cell Sci. 125, 155-165 (2012).

83. F. Motahari, A. E. Carlsson, Thermodynamically consistent treatment of the growth of a biopolymer in the presence of a smooth obstacle interaction potential. Phys. Rev. E 100, 042409 (2019).

84. S. Yamashiro et al., Myosin-dependent actin stabilization as revealed by singlemolecule imaging of actin turnover. Mol. Biol. Cell 29, 1941-1947 (2018).

85. Y. Aratyn-Schaus, P. W. Oakes, M. L. Gardel, Dynamic and structural signatures of lamellar actomyosin force generation. Mol. Biol. Cell, 22, 1330-1339 (2011).

86. T. Oliver, M. Dembo, K. Jacobson, Separation of propulsive and adhesive traction stresses in locomoting keratocytes. J. Cell Biol. 145, 589-604 (1999).

87. K. Keren et al., Mechanism of shape determination in motile cells. Nature 453,475 480 (2008).

88. D. Shao, H. Levine, W.-J. Rappel, Coupling actin flow, adhesion, and morphology in a computational cell motility model. Proc. Natl. Acad. Sci. U.S.A. 109, 6851-6856 (2012)

89. P. Maiuri et al, Actin flows mediate a universal coupling between cell speed and cell persistence. Cell 161, 374-386 (2015).

90. K. Hennig et al., Stick-slip dynamics of cell adhesion triggers spontaneous symmetry breaking and directional migration of mesenchymal cells on one-dimensional lines. Sci. Adv. 6, eaau5670 (2020).

91. N. Wang et al., Cell prestress. I. Stiffness and prestress are closely associated in adherent contractile cells. Am. J. Physiol. Cell Physiol. 282, C606-C616 (2002).

92. T. R. Kießling et al., Analysis of multiple physical parameters for mechanical phenotyping of living cells. Euro. Biophys. J. 42, 383-394 (2013).

93. M. Dembo, Y.-L. Wang, Stresses at the cell-to-substrate interface during locomotion of fibroblasts. Biophys. J. 76, 2307-2316 (1999)
94. V. Vogel, M. Sheetz, Local force and geometry sensing regulate cell functions. Nat Rev. Mol. Cell Biol. 7, 265-275 (2006)

95. R. A. Jannat, M. Dembo, D. A. Hammer, Traction forces of neutrophils migrating on compliant substrates. Biophys. J. 101, 575-584 (2011)

96. L. A. Smith, H. Aranda-Espinoza, J. B. Haun, M. Dembo, D. A. Hammer, Neutrophil traction stresses are concentrated in the uropod during migration. Biophys. J. 92, L58-L60 (2007).

97. U. S. Schwarz, R. D. Jme Soiné, Traction force microscopy on soft elastic substrates: A guide to recent computational advances. Biochim. Biophys. Acta Mol. Cell Res. 1853, 3095-3104 (2015)

98. K. A. Beningo, M. Dembo, I. Kaverina, J. Victor Small, Y.-I. Wang, Nascent focal adhesions are responsible for the generation of strong propulsive forces in migrating fibroblasts. J. Cell Biol. 153, 881-888 (2001).

99. A. Upadhyaya, J. R. Chabot, A. Andreeva, A. Samadani, A. van Oudenaarden, Probing polymerization forces by using actin-propelled lipid vesicles. Proc. Natl. Acad. Sci. U.S.A. $100,4521-4526(2003)$

100. K. A. Beningo, K. Hamao, M. Dembo, Y. I. Wang, H. Hosoya, Traction forces of fibroblasts are regulated by the Rho-dependent kinase but not by the myosin light chain kinase. Arch. Biochem. Biophys. 456, 224-231 (2006)

101. A. Ponti, M. Machacek, S. L. Gupton, C. M. Waterman-Storer, G. Danuser, Two distinct actin networks drive the protrusion of migrating cells. Science 305, 1782-1786 (2004).

102. E. Evans, Probing the relation between force-lifetime-and chemistry in single molecular bonds. Annu. Rev. Biophys. Biomol. Struct. 30, 105-128 (2001).

103. M. Vicente-Manzanares, J. Zareno, L. Whitmore, C. K. Choi, A. F. Horwitz, Regulation of protrusion, adhesion dynamics, and polarity by myosins IIA and IIB in migrating cells. J. Cell Biol. 176, 573-580 (2007)

104. C. A. Wilson et al., Myosin II contributes to cell-scale actin network treadmilling through network disassembly. Nature 465, 373-377 (2010).

105. Y. Cai et al., Nonmuscle myosin IIA-dependent force inhibits cell spreading and drives F-actin flow. Biophys. J. 91, 3907-3920 (2006).

106. S. Even-Ram et al., Myosin IIA regulates cell motility and actomyosin-microtubule crosstalk. Nat. Cell Biol. 9, 299-309 (2007).

107. A. M. Pasapera, I. C. Schneider, E. Rericha, D. D. Schlaepfer, C. M. Waterman, Myosin II activity regulates vinculin recruitment to focal adhesions through FAK-mediated paxillin phosphorylation. J. Cell Biol. 188, 877-890 (2010).

108. C.-H. Lin, P. Forscher, Growth cone advance is inversely proportional to retrograde F-actin flow. Neuron 14, 763-771 (1995) 